\title{
A Watershed for Qualia: Marc Champagne's Unified Theory of Consciousness
}

\author{
Jamin Pelkey \\ Ryerson University
}

Review of Consciousness and the Philosophy of Signs: How Peircean Semiotics Combines Phenomenal Qualia and Practical Effects, by Marc Champagne (Cham: Springer, 2018), $\mathrm{x}+127$ pp.

\section{Overview}

M arc Champagne's new book Consciousness and the Philosophy of Signs is a triumph. The book is eminently well informed, well reasoned, well written, and well worth reading. It may also still be well ahead of its time, but my guess is that it will eventually prove to be a watershed. The watershed in question concerns many important distinctions-none more important than "Qualia": the intrinsic feel of a given experience. The notion (and stubborn presence) of felt, qualitative experience has proven to be a wretched stumbling block throughout the course of the western tradition; so making progress on the problem-especially when working within the contemporary Anglo-American tradition-is not a job for the cavalier. One must tread lightly, with disciplinary grounding and respect, or be dismissed out of hand. On the other hand, an abundance of critical scea $d w \bar{i} s n e s^{1}$ is called for, some of which will inevitably cut against the grain of philosophical commitments and academic fashion.

1 The Old English term sceädwīsnes is a colorful compound (or "kenning") that can be glossed 'dividing-wisdom'. The term translates to 'reason' or 'discernment', and its two morphemes are cognate with "shed" $(<s c e \bar{a} d)+$ "wiseness" $(<w \bar{i}$ snes $)$. Notably, the historical semantics of "sceād" in the first half of the compound have nothing to do with a toolshed and everything to do with a watershed. Someone who reasons well, in other words, practices discernment or wisdom in the identification and application of separations, distinctions and divisions. 
Written in dialogue with current trends in cognitive science and analytic philosophy of mind, the book opens by affirming an influential division between Qualities and Functions in recent theories of cognition. These concepts map onto "phenomenal consciousness" and "access consciousness", respectively (following Block 1995). As Champagne clarifies up front (2018:3), this basic division has nothing to do with the infamous (and dubious) mind-body split inherited from Descartes. Rather, the distinction identifies a fundamental difference between Feeling and Doing - the former being phenomenological, the latter psychological (2018: 11). Notably, cognitive science has lavished attention on the functional/ psychological aspects of consciousness while neglecting feeling or felt qualities. Analytic philosophers of mind, in turn, have generally dispensed with felt experience, often dismissing it as an illusion, due to its practically inconsequential nature. Nonetheless, key thinkers such as David Chalmers, working at the intersection of philosophy and cognitive science, have for more than two decades called for the development of a "fundamental theory of the conscious mind" (1996) - one that integrates quality and function (Champagne 2018: 8).

In seeking to realize Chalmers' project, Champagne appeals to further distinctions and developments in pragmatism and semiotic philosophy. In so doing, his work is closely aligned with that of Mark Johnson (e.g., 2007), though this connection is not registered in the book. Both thinkers draw on pragmatist thought to illustrate how qualia should be granted pride of place in a unified theory of mind. Both insist that thinking and feeling are fundamentally integrated activities (instead of being fundamentally discrete: see Champagne 2018: 8; Johnson 2007: 1). Both argue forcefully that we must move beyond the philosophy of language to develop a more comprehensive theory of meaning. But, whereas Johnson blends the thought of William James and John Dewey with quasi-semiotic developments from Cognitive Linguistics to make his case, Champagne goes to the source-tracing both Pragmatism and Semiotic to their original integration in the work of Charles Sanders Peirce and building his case from there. This makes sense. Not only was Peirce the mentor of both James and Dewey and the acknowledged father of philosophical pragmatism, but, as Champagne clarifies (2018: 14, citing Kilpinen 2008: 217), given the scope and depth of his contributions, we can no more work to develop a non-Peircean semiotic today than we can attempt to develop a non-Darwinian biology or a non-Einsteinian physics.

Working across the span of seven well-conceived, tightly integrated chapters, Champagne makes a case for the re-evaluation and application 
of three key concepts from the hard-won thought of Peirce, arguing that grafting these concepts into contemporary cognitive science and philosophy of mind would enable the realization of Chalmers' mandate for a fundamental theory of consciousness. These three concepts are Iconicity, Tone, and Prescission (as described in more detail below). But as seasoned semioticians know all too well, the piecemeal application of Peircean ideas simply won't do. In fact, the overarching irony of Champagne's argument is that the very neglect of these three concepts in contemporary AngloAmerican thought is demonstrably the result of haphazard applications of other Peircean ideas that were taken out of context to start with-to the point of eventually becoming dissociated from Peirce altogether. "Indexicality" was adopted but divorced from "Iconicity". "Type and Token" were adopted but divorced from "Tone". "Qualia” was adopted but divorced from "Prescission". As a result, all three became relatively empty signifiers to be filled by pre-conceived habits of thought. With this in mind, it would not be far fetched to say that in writing this book Champagne is calling for something of a grand conceptual reunion (accompanied by intellectual acknowledgement and theoretical reconciliation).

To actually reap the benefit of Peircean semiotic concepts (especially the insight and clarity of thought that they enable), it is necessary to appreciate their situation relative to a broader network of relations within the overall system Peirce defines - the least dispensable aspect of which is a set of three interdependent yet irreducible categories: Firstness (Quality), Secondness (Reaction), and Thirdness (Mediation). Champagne's critical appreciation of Peirce's system is complemented by thorough explanation of its importance for contemporary Anglo-American thought. The ideas and their connections do not come easy since they cut against the grain of entrenched habits of mind. In view of such a challenging task, then, it is fortunate that Champagne marshals qualities of communication that are intelligible and straightforward, being amply illustrated with examples and woven together with narrative contextualization. I will return to an evaluation of the book's argument and approach toward the end of this review. First it will be more fitting (and hopefully more helpful) for me to offer an overview of (or take on) the book's argument itself. In so doing, I follow Champagne's lead to focus on the indispensable role that Iconicity, Tone, and Prescission should play in a fundamental theory of consciousness, paying special attention to the relationships these share with the embattled concept of Qualia.

The term "Qualia" and its companion "Quale" are so familiar to many by present day that they might easily be taken for granted. In fact, both 
terms were coined by Peirce, as Champagne discusses at length. Remarkably, then, three of the most substantial problems perceived to beset contemporary theories of Qualia would be neutralized if the concept in question were recontextualized within the larger system of thought that gave birth to it. The first of these problems can be neutralized by understanding the relationship between Qualia and "Prescission".

\section{Prescission and Qualia}

To understand prescission and its relationship to Qualia, it is first necessary to pay attention to the categories of experience. Qualia (and Quale) are by nature phenomena of fundamental Firstness in Peirce's system of thought. Any felt experience, however subtle, complex, or arresting, is first and foremost a firsthand experience: i.e., an experience of Firstness. But as soon as we reflect on such feelings, or even begin to pay attention to them, what we notice becomes inextricable from its physical effects. This necessarily introduces aspects of Secondness. If we happen to have a term for the feeling in question, this, in turn, necessarily introduces aspects of culture, language, and conventionality-modes of Thirdness-into the experience. So one of the immediate problems with discussing Qualia, which is ultimately pure Firstness, is that it is virtually impossible to isolate without recourse to Secondness and Thirdness. Even the act of naming qualia "Qualia" corrupts the phenomenon itself by introducing a concept: something "wedded to our conceptual apparatus" (2018: 119). This causal and conceptual interference might even lead us to the conclusion that what we are discussing is actually illusory. We might even despair that whatever it is, it can never be captured by science and is therefore unlicensed by reason. Can we then reasonably discuss "a concept wholly based on attention to a quality" (2018: 119)?

These would seem to be crippling defeaters; so why was Peirce undaunted by them? Champagne identifies "Prescission" or "Prescissive Abstraction" as the reason. As Champagne notes, prescision is a kind of "deliberate myopia" (2018: 5): "Prescission is what happens when, going against the facts, we suppose that some portion of a complex phenomenon is absent" (2018: 44). Prescission is a foundational practice that underlies Peirce's broader semiotic philosophy. Peirce adapts the idea from Duns Scotus's contrast between "formal distinctions" and "real distinctions" (2018: 118). The difference between one color and another is a real distinction, but the difference between the color of paint and the paint itself is a formal or "prescinded" distinction. Hence the activity of prescission is the identification of "a mere logical possibility" (Champagne 2018: 119; 
1903: EP2.294); but this does not make the possibility or the activity that generates it unreasonable or irrelevant.

It may seem bizarre that we can, for instance, imagine the smile of the Cheshire cat without the cat being present, but the fact remains that we can (2018: 46); so we need to be able to account for this ability and incorporate it into our theory building. In fact, prescission is something we do constantly, with important benefits and consequences. Any given experience, event, or object is so heavily situated and complex that we inevitably discuss its features or qualities as formal abstractions. But this does not mean that prescission is the same as decontextualization or reification: "A color like orange can be prescinded but orange does not exist apart from orange things" (2018: 21, 26).

We rely on prescissive abstraction whenever we self-reflect, whenever we notice a pattern, and whenever we reason according to a pattern (a.k.a. "diagrammatic thinking"; 2018: 105, 107). But, according to Peirce, as Champagne notes, prescission can be explained without reliance on introspection (2018: 39-40). To see how this is so, it is helpful to note that complexity subsumes simplicity (2018: 105). This points to a fundamental asymmetry between the two $(2018: 105,121)$ : i.e., complexity implies simplicity but simplicity does not imply complexity. Put differently, a relatum can exist without a relation; but a relation cannot exist without relata (2018: 12). The same holds true for Qualia: Access Consciousness (Function) implies the presence of Phenomenal Consciousness (Qualia), but Phenomenal Consciousness does not imply the presence of Access Consciousness. Hence, there is something that Qualia, Relata and simplicity share in common. In Peircean terminology, this common ground can be described as "Tone".

\section{Tone and Qualia}

To appreciate the role that Tone plays in the validation of Qualia, and in the realization of Qualia's integration with Function, it is helpful to clarify its status relative to two key Peircean triads: (1) the Representamen-ObjectInterpretant triad and (2) the Tone-Token-Type triad.

First consider Qualia's relationship to the three elements of any triadic sign relation. As Champagne describes, "despite the unbreakable triadic bond which characterizes any representation, whatever is burdened with the logical duty of standing for something else-no matter what it may consist in-can be prescinded in such a way as to disregard its employment in that capacity" (2018: 36). Any sign relation must involve thee elements: (1) a representamen (or sign vehicle), (2) an object (being represented), 
and (3) an Interpretant (i.e., effective creation of a new sign in the mind of an interpreter, whether witting or unwitting). Tone identifies a sign vehicle (or "Representamen") involved in any act of prescission. It is "a monadic dimension that has the power to be the qualitative vehicle of representation but which in virtue of its ordinal primacy remains serenely ignorant of whether it is actually employed in so raucous an activity" (Champagne 2018: 38; Peirce c.1896: CP 1.422-426).

While the Peircean distinction between Type and Token has proven to be very influential in the analytic tradition, the curious deletion of Tone from the picture undermines the effectiveness (and validity) of the distinction. Since all tokens subsume tones (2018: 39), and since "a type is what permits one to encounter tokens of the same tone" (2018: 65), any attempt to accurately apply Type and Token without reference to Tone, will simply miss the point and fumble opportunities to solve problems that are otherwise intractable-prominently including the hard problem of consciousness.

Applied to the problem of Qualia, or phenomenal consciousness, Champagne notes that "It is not that access-consciousness and phenomenal-consciousness are tokens of different types; rather, the latter is the tone of the former's tokens" (2018: 39). In other words, felt experience serves as the tone of all token instances of functional experience. This approach is important since it circumvents mere reification of prescinded features. And by "refusing to reify the features it prescinds, such an approach allows us to respect the distinct character of phenomenal experience without turning it into a chimera" (2018: 38-39).

While indexical relations are enabled by tokens, tones serve to make iconic relations possible. If the relationship between a sign vehicle and its object is iconic, as Champagne clarifies, the sign vehicle must be a tone: "only as a token can a sign-vehicle have an indexical bond to its object. Similarity requires a shared quality, so only as a tone can a sign-vehicle enjoy an iconic bond to the quality referred to" (2018: 58). With this in mind, we begin to see the overlooked importance of iconicity (relationships of similarity or resemblance) for framing an adequate account of Qualia and the indispensable role it plays in consciousness.

\section{Iconicity and Qualia}

Iconicity concerns both mind-world (observed) similarities and worldworld (inherent) similarities between sign-vehicles and their objects (2018: 57). Taking color as an illustration, Champagne argues (following much more careful discussion than I am able to reproduce in a brief book re- 
view) that "experiences are red in the same sense in which apples are red" (2018: 73). Given the symmetrical nature of resemblance, if perceptual experience A resembles apple color B, it is also the case that apple color B resembles perceptual experience $A$. Hence, in true iconic relations, only an Interpretant can determine which of the two relata is a sign vehicle and which is an object (2018: 61). Similarities shared between A and B are also necessarily similarities between features of A and B, otherwise the two would be indistinct. As such, iconic signs must be said to share underlying Tones (instead of an underlying Tokens), enabling features to be prescinded from the complex whole (2018: 62).

These distinctions and definitions are important to note since mainstream philosophy of language (which tends to inform cognitive science and philosophy of mind) is overwhelmingly focused on indexical sign functions (2018: 63). Indexicality involves relations of direct reference and efficient causality. But causality and direct reference only serve to describe aspects of functional consciousness. Qualitative consciousness cannot be captured by either. In Champagne's words, "Since qualia are not captured by causality and since indexicality works precisely by exploiting causality, qualia are not captured by indexicality" (2018: 53).

Iconic relations, on the other hand are perfectly suited for referring to qualia, and the more iconic the relation, the more a given sign vehicle and its object will resemble each other (2018: 84). As perceptions of resemblance increase, felt sensations become dominant, and degrees of coalescence between sign vehicles and their objects increase (2018: 83-85). This is typified in the contemplation of a complex visual icon such as a painting or film, in which we are often able to lose ourselves as coordinated cascades of sign vehicle tones and their iconic objects coalesce in consciousness (2018: 85).

Mapping iconic relations to phenomenal/qualitative aspects of consciousness on one hand and indexical relations to material/functional aspects of consciousness on the other enables a unified account of consciousness, but it does not obliterate the border between them. The gap between the material and the phenomenal remains, and it remains at both epistemic and ontological levels (2018: 52, 79-87, including helpful discussion of a related distinction in Poinsot 1632). Notably, the bridge between the two is neither iconic nor indexical. The bridge between the two is a more general semiotic system: Champagne's account demonstrates that a general (Peircean) semiotic actually allows for a unified account that is both licensed by reason and resistant to reification. 


\section{Further Evaluation}

As mentioned above, I find Champagne's argument and approach to be a triumph of clear writing, with an abundance of helpful examples, and a refreshing knack for narrative contextualization. Unlike much other work in semiotics, Champagne also goes to great lengths to accommodate readers who are unfamiliar with semiotic terminology. The flip side of such accommodation might be overcorrection in some cases, potentially undermining the accurate communication (or didactic explication) of new ideas in the process. To be clear, I notice very few cases of such overreach in my reading of this text but would like to point out a handful in case they prove helpful for future editions or subsequent developments of the project.

First, given the importance of tracking the categories for Peircean semiotic, and given the ethics of terminology related to core concepts in any system, there are cases in which the ordering and/or paraphrase of key terms from Peirce could be handled more carefully, lest alternative uses obfuscate more than they clarify. Take, for example, the cloaked reference to iconicity, indexicality, and symbolicity in the following passage: "According to the Peircean theory I defend, there are three ways in which one can know things, namely by convention, causality, and similarity" (2018: 52). This listing is paraphrased and also reverse-ordered relative to Peirce's system. These decisions in general may be well and good (as Champagne himself argues elsewhere, 2015: 541-544); but, in such cases, clarification of terms relative to Peirce's system-either in a footnote or by using intext parentheticals-would be helpful to ensure the connections are not lost by readers who are still learning to make them. The same terms are used on page 5, again without didactic mapping, although the ordering is reversed to match Peirce's system of categories: i.e., "similarity, causation, and convention". On page 93, the Peircean terms are used instead ("symbols, indices, and icons"), but the ordering is reversed yet again relative to Peirce's system. On the facing page (2018: 92), the three sign functions are clarified in-text using parenthetical asides, and the ordering is still reversed. Taking more care with consistent ordering and clarification in such instances might serve readers even better. ${ }^{2}$

Next, considering terminology, it is sometimes necessary, in spite of approachability concerns, that key terms should induce struggle-in order

${ }^{2}$ Again, to clarify, this is not to deny that reverse-ordering may well be more didactically and experientially meaningful - a point that Champagne argues elsewhere (2015: 541-544). Rather, the more basic issue at stake is consistency of presentation with explicit acknowledgement of rationale in situ. 
to change old habits of mind. Champagne seems amply aware of this point in most cases. One exception might be his use of "interpretation" in place of the less familiar "interpretant" in the text (see, e.g., 2018: 4, 79). The former is certainly more approachable, and as passages elsewhere in the text make clear (see e.g., 2018: 61, 81, 92), Champagne is well aware of the difference. The Peircean Interpretant goes beyond mere acts of interpretation to include the creation of a new sign, typically mediated by various degrees of habit or preparation such as biological endowment, populationlevel enculturation, and individual and group-level training-enabling an individual to interpret across a range of modalities from the deliberate to the instinctive. Clarifying this more directly might serve readers well.

Finally, it might be helpful to reconsider citation practices for source dates that refer to posthumously published works. This is especially important for tracking insights from Peirce-and even moreso when trying to rehabilitate and apply mature ideas from Peirce. In such cases, citation dates that are not historical risk making one's argument more difficult to evaluate. Although, to be clear, the intention here appears to be reader accommodation, certainly not deliberate obfuscation.

Again, these are minor suggestions indeed compared to the rigor, empathy, and attention that Champagne marshals throughout the text in the production of this book. Helpful turns of phrase interweave with crystal clear argumentation and refreshing shifts to illustrative examples and narrative historiography. In the process we find excellent overviews of semiotics (2018: 14-27) and chart the inception and demise of key terms borrowed from Peirce by Anglo-American philosophers. In the process we also discover that Champagne is remarkably conversant with contemporary cognitive science and analytic philosophy (both historical and contemporary). And in spite of his equally strong grasp of Peircean pragmatism and Peircean semiotic, we also find a thinker who is not susceptible to narrow-minded or preachy polemics (see, e.g., 2018: 59-60). All three traditions are taken seriously and treated with respect in a spirit of cooperation toward a shared goal. Even so, when the time comes to reject invalid ideas or call out the absurdity of illegitimate conclusions, Champagne pulls no punches. In the process, he does more than offer a new vision for unified consciousness (which would certainly be enough). He also models a promising vision for the future of semiotics as a perspective on the disciplines, prominently including philosophy and the other cognitive sciences. 


\section{Semiotic Vision}

Champagne's vision for semiotics could be described as "Peirce-Plus": an approach that is deeply acquainted with Peirce's semiotic architectonic while still managing to be ecumenical (2018: 7). He insists that there is ultimately no non-Peircean semiotic (as discussed above), while also insisting that not all semiotic is Peircean (2018: 14). Accordingly, Champagne's vision integrates semiotics with pragmatism (e.g., 2018: 7; see also Champagne 2016) while also taking discipline-specific issues and debates seriously. He manages his argument both as a semiotician and as a disciplinary insider. More to the point, in applying "philosophy of signs to philosophy of mind" (2018: 13), Champagne casts a long-term vision for the reorientation of all disciplines toward a general semiotic as a fundamental theory if not a new science (2018: 8). This can be seen as a special priority for disciplines that have adopted key concepts from semiotics without taking care to appreciate and apply them systematically. In the case of Qualia, the term's dissociation from its Peircean origins and its accompanying conceptual apparatus-along with other bastardizations such as the dissociation of Tone from Token and Type-have had dire consequences (as Champagne illustrates throughout the book). Such neglect creates seemingly intractable dilemmas and distractions where there might otherwise have been no real problems to speak of.

Champagne's vision for semiotics is one that is awake to history (building on, e.g., Deely 2001), aware that signs are relational instead of material (2018: 19-20), and keenly aware of related fallacies-such as that of "limiting signs to language and human culture" (2018: 15). Insights such as these enable him to argue that the philosophy of mind must be broader than the philosophy of language, since language simply cannot convey everything $(2018: 63,68)$. The fact that Iconicity is still largely absent from formal semantics (2018: 7), in spite of the crucial role it plays in referring to Qualia; the fact that Tone was neglected as a ground for prescission; and the fact that contemporary Anglo-American thought remains averse to Qualia (in spite of its pervasive presence), all point to a fundamental need for cultivating appreciation for the role of Firstness. Firstness, Champagne affirms, is necessarily ineffable. It evades description even though it pervades our experience (2018: 71; following Short 2006: 171). Since semiotics is "a paradigm that explicitly includes Firstness in its basic commitments" (2018: 120), in an account that is already fully integrated with function, semiotic philosophy provides a "conceptual apparatus" that makes qualia seem sensible (2018: 117). 
Finally, Champagne's vision for semiotics is one that works to cultivate "a worldview in which meaning does not seem out of place" (2018: 110). Indeed, only in a "disenchanted vision of the world" (2018: 96) could qualities of Firstness seem suspicious-especially given that such qualities are the norm. This points to a veritable stockpile of "revisionist work" that is needed in order to move Anglo-American thought (and other varieties of western thought) away from a "fundamentally mistaken worldview" $(2018: 89,91)$. In the process, it helps to remember that progress is possible: Champagne reminds us that even discussions of consciousness were once taboo in this tradition (2018: 2). His argument for the reality of Qualia and its need for conceptualization via "prescissive intervention" (2018: 113) are all the more compelling in this light. And the narrow-minded neglect that led to the exclusion of prescission, Tone, and Iconicity-due to the illegitimate alienation of Qualia and Indexicality from semiotic thought-must be relentlessly called to task.

Cognitive science and philosophy of mind now have a viable account of phenomenal consciousness qua "Qualia" that is not only licensed by reason but also fully integrated and reconciled with oppositional accounts of access consciousness. As a result, the argument and evidence in Champagne's book, once acknowledged, are sure to lead philosophy of mind and cognitive science in a fresh, new direction.

\section{References}

BLOCK, Ned.

1995. "On a Confusion about a Function of Consciousness", Behavioral and Brain Sciences 18.2, 227-287. https://doi.org/10.1017/S0140525X00038188

CHALMERS, David J.

1996. The Conscious Mind: In Search of a Fundamental Theory (Oxford: Oxford University Press).

CHAMPAGNE, Marc.

2015. "A Less Simplistic Metaphysics: Peirce's Layered Theory of Meaning as a Layered Theory of Being", Sign Systems Studies 43.4, 523-552. https://doi.org/10.12697/SSS.2015.43.4.10

2016. "Can Pragmatists Believe in Qualia? The Founder of Pragmatism Certainly Did ...", Cybernetics and Human Knowing 23.2, 39-49.

2018. Consciousness and the Philosophy of Signs: How Peircean Semiotics Combines Phenomenal Qualia and Practical Effects (Cham: Springer). 
DEELY, John.

2001. Four Ages of Understanding (Toronto: University of Toronto Press).

JOHNSON, Mark.

2007. The Meaning of the Body: Aesthetics of Human Understanding (Chicago: University of Chicago Press).

KILPINEN, Erkki.

2008. "Memes Versus Signs: On the Use of Meaning Concepts about Nature and Culture", Semiotica 171.1-4, 215-237.

PEIRCE, Charles Sanders.

i.1867-1913. Collected Papers of Charles Sanders Peirce, Vols. 1-6 ed. Charles Hartshorne and Paul Weiss (Cambridge, MA: Harvard University Press, 1931-1966); Vols. 7-8 ed. Arthur Burks (same publisher, 1958). Cited as CP.

i.1893-1913. The Essential Peirce: Selected Philosophical Writings, Vol. 2, ed. Peirce Edition Project (Bloomington: University of Indiana Press, 1998). Cited as EP2.

POINSOT, John.

1632. Tractatus de Signis: The Semiotic of John Poinsot, ed. John Deely, trans. John Deely and Ralph Austin Powell (2nd ed., South Bend, IN: St. Augustine's Press, 2013).

SHORT, Thomas L.

2006. “Response to Carl Hausman”, Recherches sémiotiques/Semiotic Inquiry 26.2-3, 157-176. 